\title{
Graphene-Coating for Efficient Electronics Cooling
}

\author{
Created by: Maher Al-baghdadi ${ }^{1}$
}

1, Kufa Centre for Advanced Simulation in Engineering (KCASE), Department of Mechanical Engineering, Faculty of Engineering, University of Kufa, Iraq.; mahirar.albaghdadi@uokufa.edu.iq

Version received: 19 July 2020

check for updates

\begin{abstract}
Thermal management is essential in electronics, as it improves reliability and enhances performance by removing heat generated by the devices. Thermal management of handheld systems such as laptops is becoming increasingly challenging due to increasing power dissipation. The power dissipated per unit area on the laptop electronic chips is increasing while the area of the chips itself it decreasing, resulting a high heat flux that causes an increase in temperature. The increasing temperature adversely affects the performance of laptops and in many cases leads to failure through such modes as thermal fatigue and dielectric breakdown. In this work, three dimensional steady state CFD model of a laptop motherboard is presented. The model accounts for heat transfer for both natural convection and radiation to the ambient air temperature. The present CFD study allow accurate, rapid, physical modelling to make decisions on materials, components and layout beside power control feedback to achieve performance and target lifetime with reduced testing requirements. An alternative design for the cooling of laptop microprocessor using only passive cooling is proposed. The results showed that the assembled a thin plate of a copper material coated with graphene and use it as a heat sinks with the microprocessor of the laptop providing an efficient and economical solution in thermal management. Considerable drop in microprocessor temperature is obtained through the heat dissipation path suggested in the new design. The proposed passive cooling solution has the advantages of fanless operation compared to the existing active cooling solutions such as the noise-free operation, lower energy consumption and higher reliability. We hope this work may open the way for huge boost in the technology of electric cooling by innovative manufacturing techniques.
\end{abstract}

\section{Introduction}

\subsection{Background}

Heat is generated in a resistive element for as long as current continues to flow through it. This creates a heat build-up and a subsequent temperature rise at and around the component. The temperature of the component will continue rising until the component is destroyed unless heat is transferred away from it. The temperature of the component will remain constant when the rate of heat removal from it equals the rate of heat generation $[\underline{1}]$.

In electronic systems, the temperature of the circuits on which there is current, increases drastically. The reason of this situation is, when the electronic current passes through the circuit, some of the kinetic energy changes into heat. Another important reason of the temperature increase in electronic circuits is current leakage. During the electron transmission between the elements of a circuit, there are usually current leakages which cause heat transfer. With the help of the improving technology, the elements of circuits became smaller in size, as a result of this, their capacitances decreased and current leakage increased. This increases the acceleration of heating in the electronic circuit. With the increased velocity of the particles, the heat that is generated due to particle vibrations increases, which in turn leads to 
more current leakage $[\underline{1}],[\underline{2}]$.

Heat that is generated in electronic systems is proportional to the square of voltage and the frequency of the system ${ }^{[\underline{1}]},{ }^{[2]}$. Because of this reason, engineers try to increase the frequency and decrease voltage to be able to decrease heat generation in order to have the technology for increased speed. The cooling of electronic equipment is a major research subject, since there are complex electronic systems in automobiles, aircraft, computers, ships, and in all other devices that are the primary components of today's life. Most of these electronic systems control crucial parts and mechanisms, which cannot tolerate mistakes. The main objective of engineers is not only to decrease the cost of goods but also to achieve high reliability and high performance in systems. The reliability, functionality and even consumer satisfaction are concerned with thermal management. Thus, many kinds of heat control technology, theory and device are developed [3] .

Electronic portable devices, especially portable computers, have become pervasive and popular nowadays. As the technology progresses and the market demands, more functions have become integrated and packed into these devices in conjunction with higher performance semiconductor chips, while the size of the devices is decreasing. The microprocessor in a personal computer has the largest power consumption of all devices and its consuming trend towards increasing. In addition, Chipset, audio, graphic processor, memory, and etc. are also beginning to generate more heat due to higher performance priority. On the other hand, there is a trend toward portable computer becoming thinner and lighter []․

There are some problems when laptop computer design. Space allocation, definition and area of printed circuit boards, heat handling, and noise interruption are the topics which determine whether laptop computers are successful or not. Among them, the heat problem plays a more important role than before. In the past, there was no overheating consideration, for the performance of microprocessor is not as high as it is now ${ }^{[\underline{5}]}$.

The thermal management system within an electronic device is design to transfer and disperse thermal energy that accumulates during operation so that the device can maintain optimal thermal performance.

Thermal management is critical to modern laptop microprocessors as their component density and power dissipation continue to increase to meet market demand and higher performance standards. Because of thermal constraints, many state-of-the-art multi-core microprocessors have to operate at lower clock frequencies than preferred when air cooled due to the hazard of overheating and thermal degradation of the device. Additionally, thermal management for laptops has gained significant attention in the last decade, as newer, smaller devices become mainstream and starts to incorporate multi-core microprocessor technology []].

Laptops using mobile microprocessors require thermal management. The term "thermal management" refers to two major elements: a cooling solution properly mounted to the microprocessor, and effective airflow through a part of that cooling solution to evacuate heat out of the system. The ultimate goal of thermal management is to keep the microprocessor at or below its maximum operating temperature. For higher power processors today, almost all laptops employ some form of an active cooling solution. This can be accomplished one of two ways. The first way is to have a heat sink attached directly to the processor. The second way would to use a remote heat exchanger []․ With limited space, and varying laptop design, layout, and processor location, laptop cooling solutions vary greatly between laptops from different manufacturers. A remote heat exchanger, offers more flexibility in thermal design, because the actual heat sink and fan can be placed far from the processor []ㅛ.

Designers of laptop computers take the double pressure of low cost and high performance on their shoulders. Usually, the failure of final layout in laptop computers is due to bad thermal considerations. 
This is because thermal aspect is always put in the last part of design []ㅡ, [ㅁ] .

Some laptop designers were used metal case in laptop computers, their results showed that the skin temperature and main heat dissipate components temperatures are decreased ${ }^{[11]}$. Other designers proposed a new component arrangement considering passive paths and using the back side of the liquid crystal display screen. A simple correlation based thermal analysis of their proposed system was presented. It is demonstrated that placing the computer processing unit, the motherboard, and the memory on the lid creates enough surface area for passive cooling [ $\left.{ }^{8}\right]$.

\subsection{Graphene}

Graphene-based composites are emerging as new class of materials that hold promise for several applications. Graphene, a single sheet of graphite, possesses extraordinary electrical, thermal, and mechanical properties arising from its unique structure. Graphene is often thought to hold advantages over other materials in electronics cooling systems because of its higher thermal conductivity. Thus, high thermal conductivity could suggest very good heat sinking and low temperature rise during device operation [12], [13], [14].

\subsection{Computational fluid dynamics (CFD)}

The increasing complexity and power density of modern electronics has challenged the traditional approach of using prototypes and testing. The modern Computational Fluid Dynamics (CFD) simulation software developed for challenging environments and high power dissipation devices has led to a

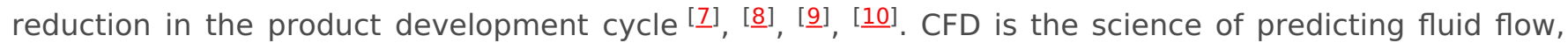
heat transfer, mass transfer, phase change, chemical reaction, and related phenomena by solving the mathematical equations that govern these processes using a numerical algorithm on a computer [피. The results of CFD analyses are relevant in: conceptual studies of new designs, detailed product development, troubleshooting, and redesign. CFD analysis complements testing and experimentation, by reduces the total effort required in the experiment design and data acquisition. CFD complements physical modelling and other experimental techniques by providing a detailed look into our fluid flow problems, including complex physical processes such as turbulence, chemical reactions, heat and mass transfer, and multiphase flows. In many cases, we can build and analyze virtual models at a fraction of the time and cost of physical modelling. This allows us to investigate more design options and "what if" scenarios than ever before. Moreover, flow and heat transfer modelling provides insights into our fluid flow problems that would be too costly or simply prohibitive by experimental techniques alone. The added insight and understanding gained from flow and heat transfer modelling gives us confidence in our design proposals, avoiding the added costs of over-sizing and over-specification, while reducing risk $[\underline{15}]$.

\subsection{Objective of this work}

Improving the efficiency of disperse heat more quickly from the laptop microprocessor is the central focus of this design research. If a new design for the cooling of laptop microprocessor can disperse heat more quickly, it is theorized that the operating temperature of the microprocessor can be lowered and the efficiency of the entire thermal management system can be improved by providing a large heat flux initially in the system.

In this work, three dimensional CFD model of a laptop motherboard with different technique of cooling has been presented. The model accounts for heat transfer for conduction through the printed-circuit with all attached electronic components materials and for both natural convection and radiation to the ambient air temperature. The cooling techniques typical in laptops will be reviewed with an overall focus on improving the efficient and economical solution in thermal management. After the analysis of the results, the objective of the study is to propose and thermally analyze an alternative design for the cooling of laptop microprocessor using only passive cooling. In an attempt to increase total heat transfer from the microprocessor to the thermal management system, a new system prototype was designed to 
have a higher total thermal conductivity than the existing system component. The best way to increase this system property was to incorporate graphene into the current system design.

\section{CFD modelling}

\subsection{Computational domain and material properties}

Motherboard from $h p^{\circledR}$ laptop company has been used as a case study in this work. The major components considered in this study included all main components of the laptop $h p^{\circledR}$ motherboard such as printed circuit board, CPU, RAM, GPU, miscellaneous ...etc., as shown in Figure 1, are modeled according to measured dimensions and manufacturers' specifications. The printed circuit board and the attached components consist of different materials. Material properties of each component are shown in Table 1.

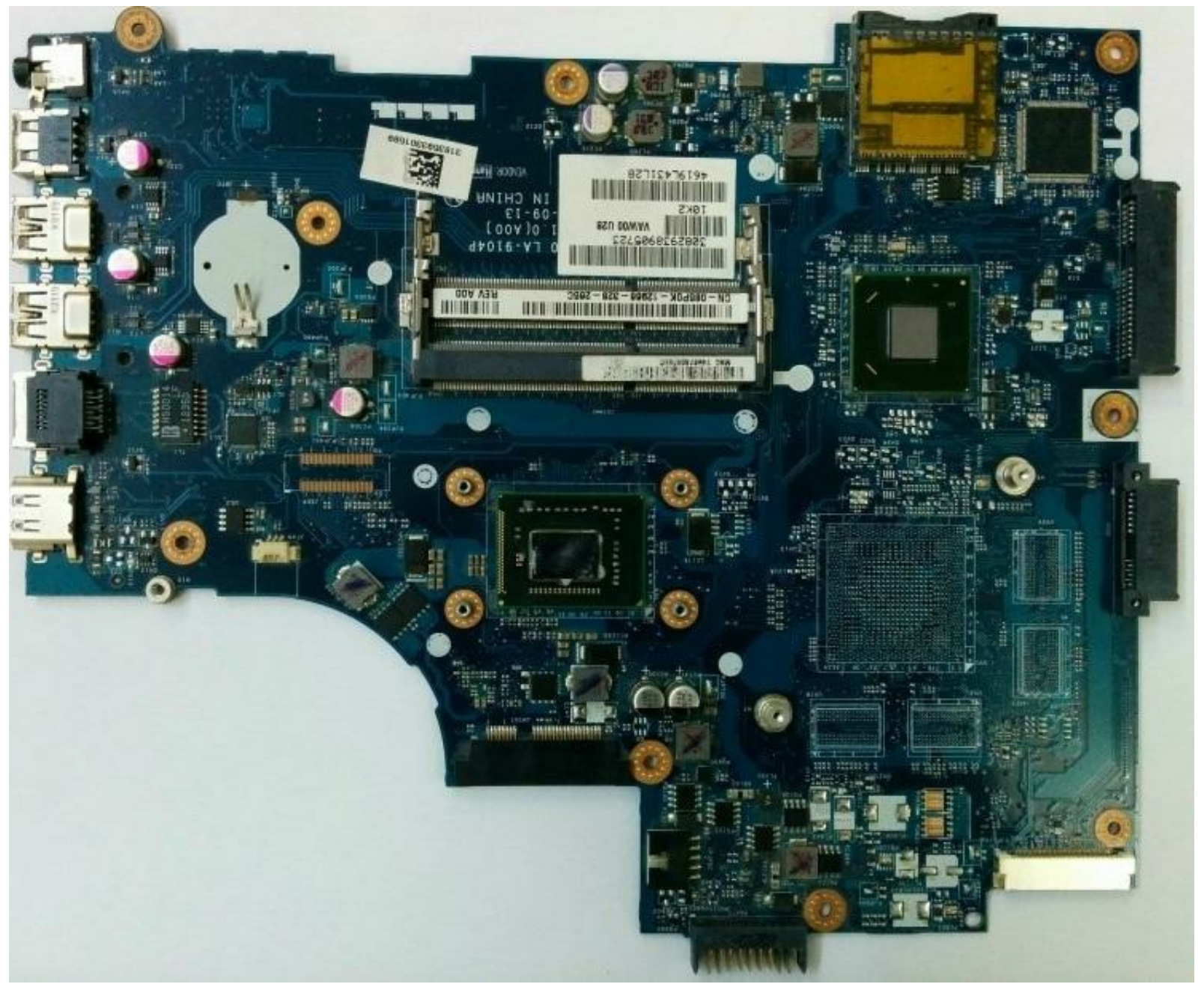

(a) $h p^{\circledR}$ motherboard. 


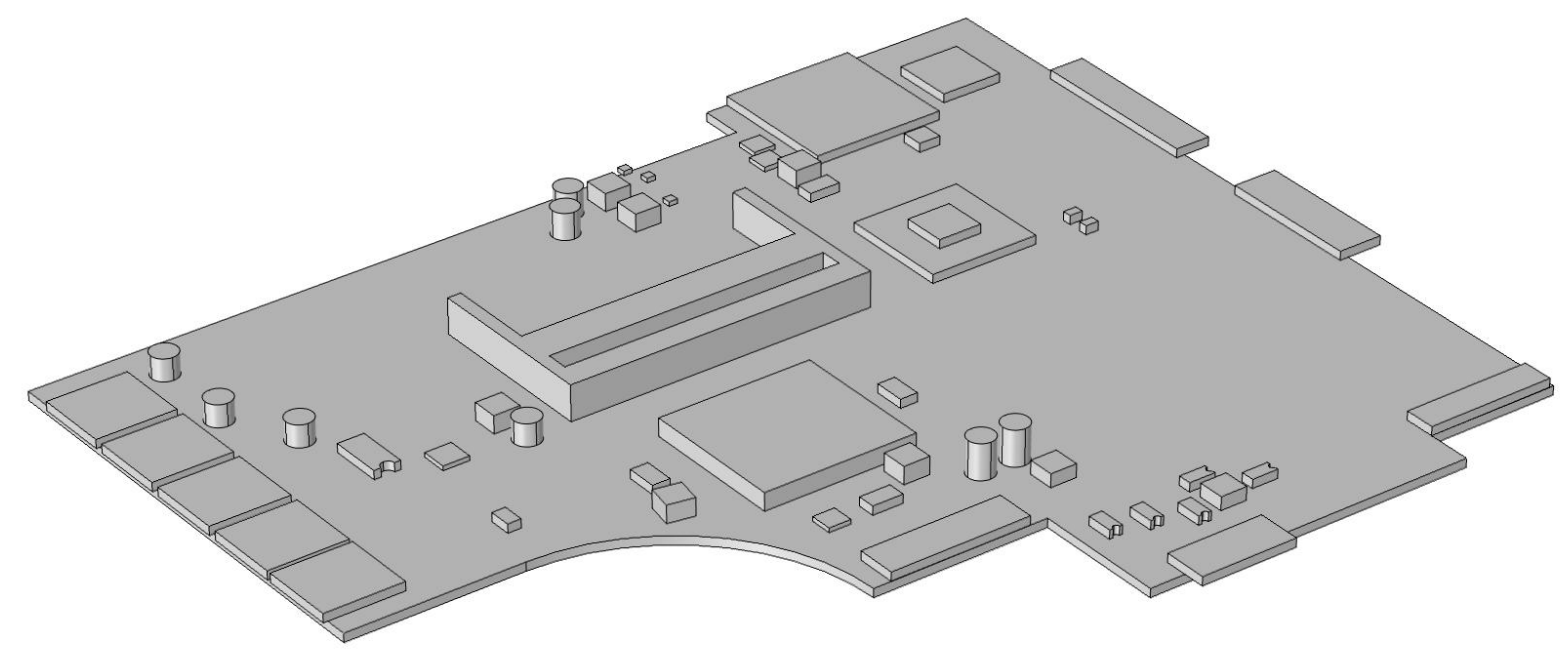

(b) three-dimensional computational domain.

Figure 1. Motherboard components of $h p^{\circledR}$ laptop company.

In a laptop computer, there is not much space left among the components that are placed very tightly. That reduces the available air inside the chassis to a level that convection cannot be the main mode of heat transfer inside the chassis. As a result, conduction plates and heat pipes become the available options to transfer heat from sources to surfaces. Fans and remote heat exchangers that are used together with heat pipe systems constitute the active paths. A remote heat exchanger, offers more flexibility in thermal design, because the actual heat sink and fan can be placed far from the processor. The heat pipe transfers heat from the microprocessor to a fin-tube type heat exchanger at the other end. The heat exchanger has a fan placed nearby it (Figure 2). Heat conduction plates that are used for heat transfer to the outer surfaces of the chassis are the passive paths for heat dissipation. 


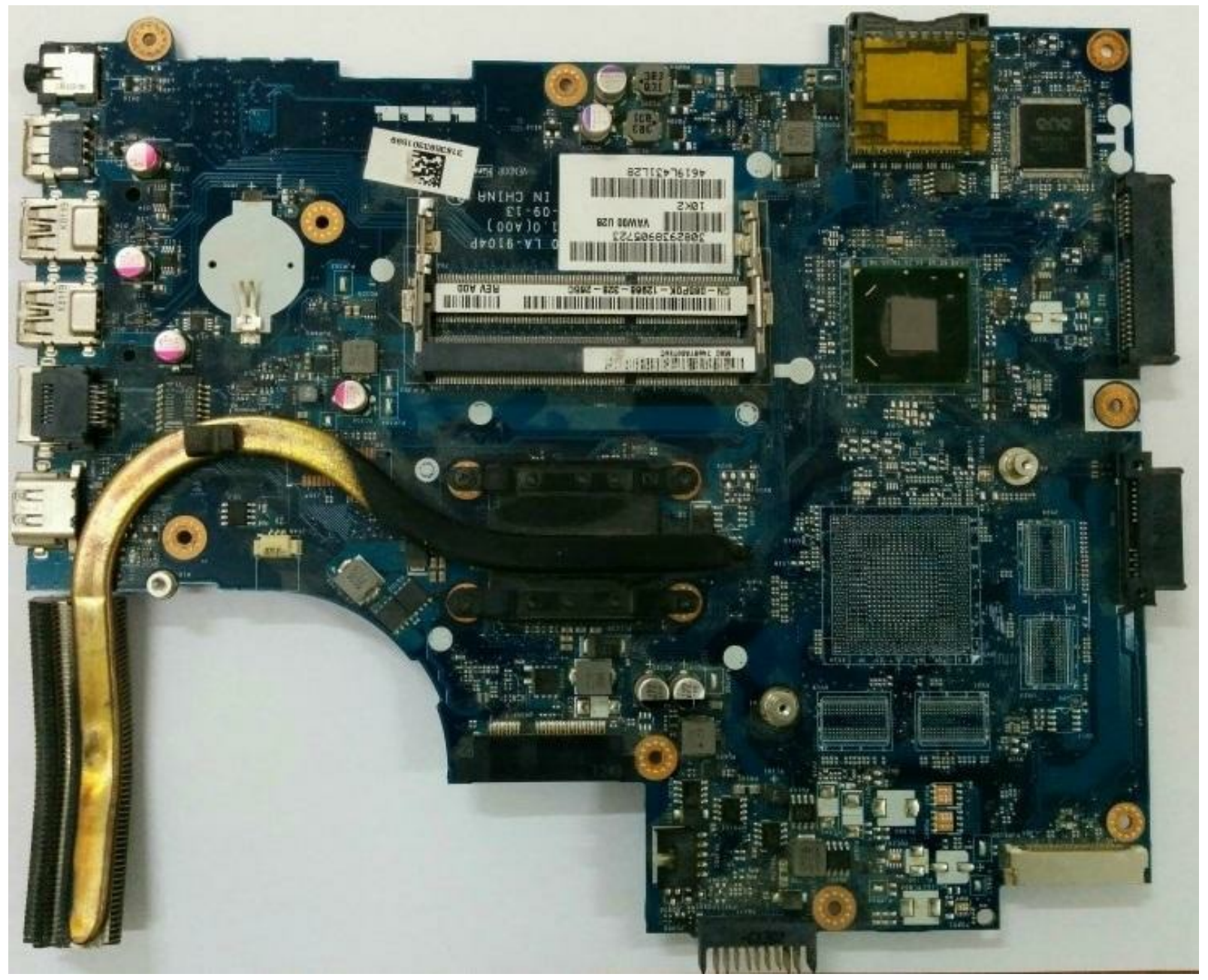

(a) $h p^{\circledR}$ motherboard with thermal management system (heat pipe).

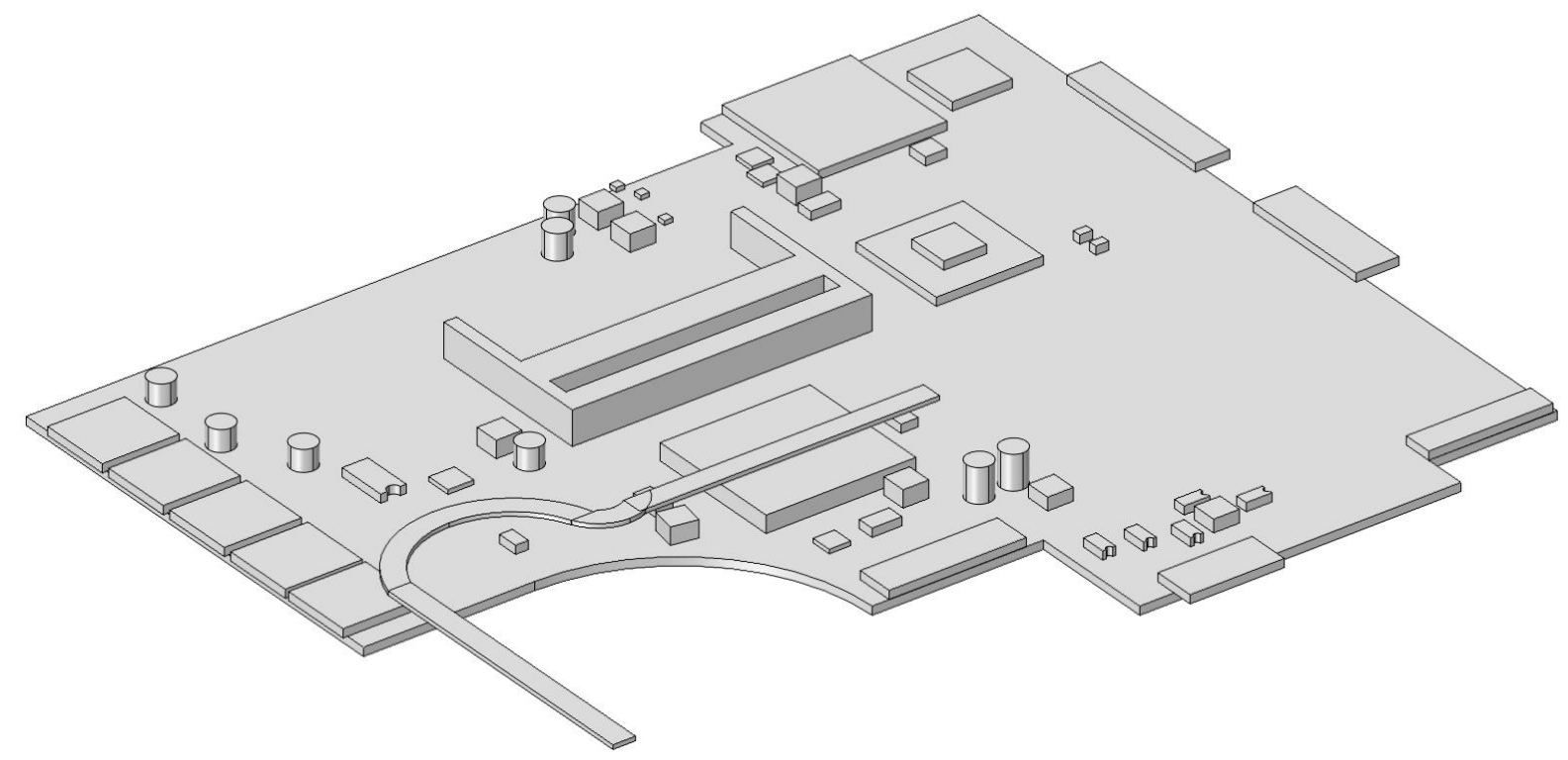

(b) three-dimensional computational domain with thermal management system (heat pipe).

Figure 2. Motherboard components of $h p^{\circledR}$ laptop company with thermal management system (heat pipe).

Table 1. Material properties. 


\begin{tabular}{lllll}
\hline Material & $\begin{array}{l}\text { Density } \\
\rho[\mathrm{kg} / \mathrm{m} 3]\end{array}$ & $\begin{array}{l}\text { Heat capacity } \\
C_{p}[\mathrm{~J} / \mathrm{kg} . \mathrm{K}]\end{array}$ & $\begin{array}{l}\text { Thermal conductivity } \\
k[\mathrm{~W} / \mathrm{m} . \mathrm{K}]\end{array}$ & $\begin{array}{l}\text { Emissivity } \\
\varepsilon\end{array}$ \\
\hline FR4 (Circuit Board) & 1900 & 1369 & 0.3 & 0.6 \\
Silicon & 2329 & 700 & 130 & 0.6 \\
Nylon & 1150 & 1700 & 0.26 & 0.6 \\
Glass & 2200 & 480 & 1.1 & 0.6 \\
Aluminum Alloy 6063 & 2700 & 900 & 200 & 0.6 \\
Copper & 8700 & 385 & 400 & 0.6 \\
Copper coated with Graphene & 8700 & 385 & 460 & 0.6 \\
\hline
\end{tabular}

\subsection{Modelling equations}

The heat transfer in a printed circuit board is take place in three ways, conduction, convection, and radiation. The temperature field is obtained by solving the energy equation [15]. Heat transfer by the conduction mode occurs when heat is transferred within a material or from one material to another. The energy transfer is postulated to occur because of kinetic energy exchange by elastic and inelastic collisions of atoms, and by electron drift. Heat energy is always transferred from a region of higher energy to an area of lower energy. The energy level, or temperature, of a material is related to the vibration level of the molecules within the substance. If the regions are at an equal temperature, no heat transfer occurs. Convection is the result of two energy transfer mechanisms: fluid motion and molecular motion. Convection is a combination of the bulk transportation and mixing of macroscopic parts of hot and cold fluid elements, heat conduction within the coolant media, and energy storage. Convection can be due to the expansion of the coolant media in contact with the device. This is called free convection, or natural convection. Convection can also be due to other forces, such as a fan or pump forcing the coolant media into motion. Radiation is the only mode of heat transfer that can occur through a vacuum and is dependent on the temperature of the radiating surface. Radiation heat transfer involves emittance, absorptance, reflectance, and transmittance at the surface of an object.

\subsection{Computational grid}

The governing equations were discretized using a finite-volume method and solved using an academic edition of multi-physics computational fluid dynamic (CFD) package. Stringent numerical tests were performed to ensure that the solutions were independent of the grid size. A computational quadratic mesh consisting of a total of 23744 domain elements, 15924 boundary elements, and 2164 edge elements for the case of absence thermal management system and a total of $=$ domain elements, $=$ boundary elements, and = edge elements for the case of presence thermal management system ware found to provide sufficient spatial resolution respectively (Figure 3). The coupled set of equations was solved iteratively, and the solution was considered to be convergent when the relative error was less than $1.0 \times 10^{-6}$ in each field between two consecutive iterations. 


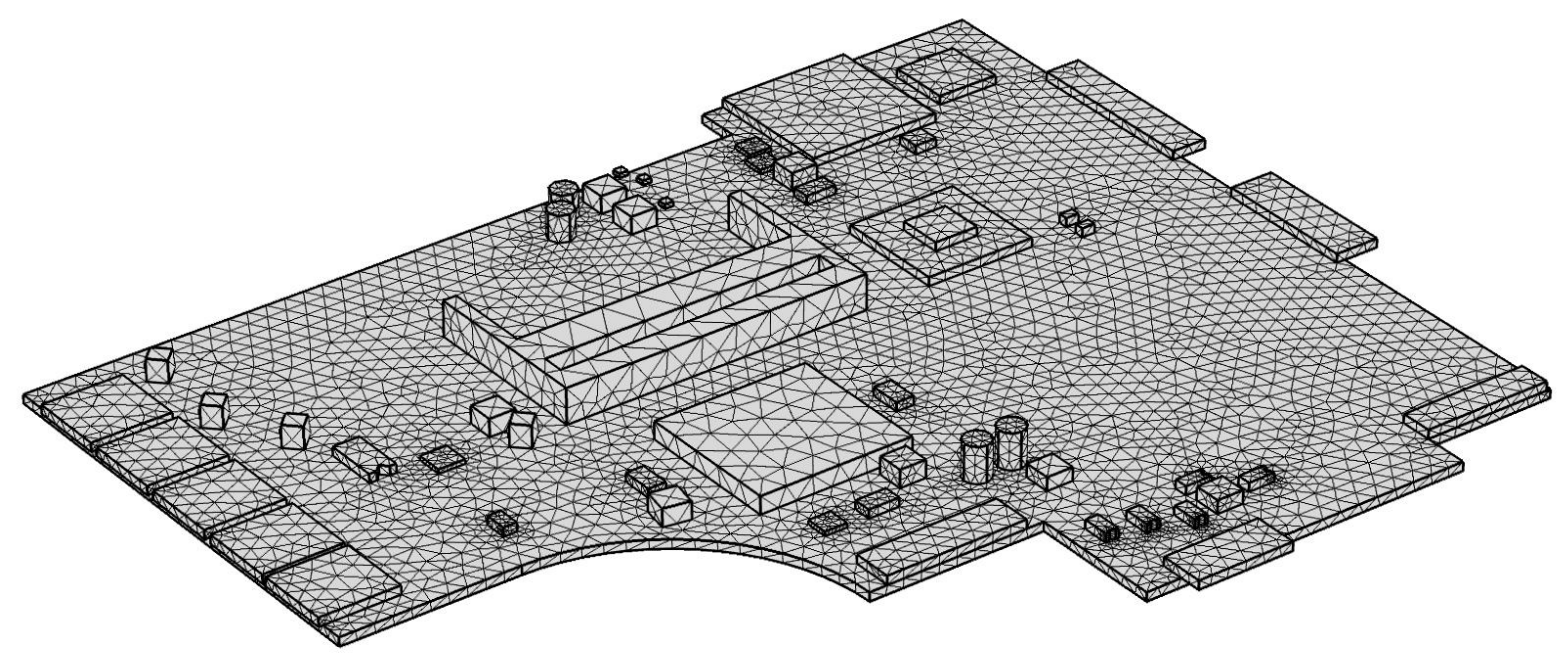

(a) without thermal management system.

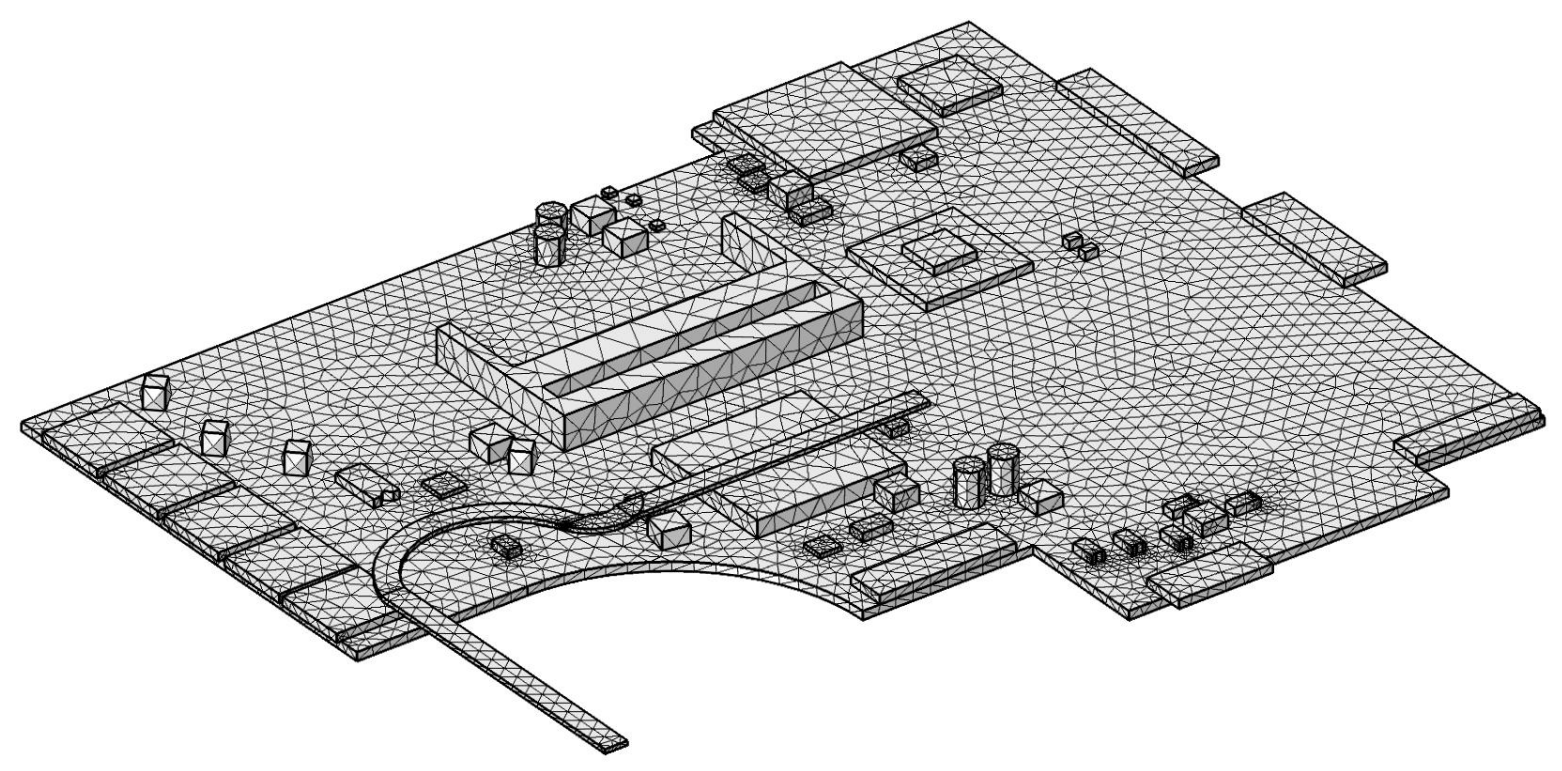

(b) with thermal management system (heat pipe).

Figure 3. Computational mesh of the computational domain (quadratic).

\section{Results}

The comprehensive three-dimensional CFD model allows for the assessment of important information about the detail of transport heat generation inside the motherboard with all its components and transfer to ambient air by convections and radiations. The ambient temperature condition is set to room reference temperature $25{ }^{\circ} \mathrm{C}$. The printed circuit board and the attached components are made from different materials as outlined in Table 1.

\subsection{Base case}

At present, single heat pipe is used in laptops as a thermal management system. The heat pipe transfers heat from the microprocessor to a fin-tube type heat exchanger at the other end. The heat exchanger has a fan placed nearby it. Heat conduction plates that are used for heat transfer to the outer surfaces of the chassis are the passive paths for heat dissipation. Figure 4 shows the temperature distribution in the laptop mother board during operating without any thermal management system and also when operating with thermal management system using copper heat pipe in the same conditions. 
The result shows a considerable reduction in microprocessor temperature when using heat pipe technique.

\subsection{New suggested thermal management systems}

Figure 5 shows the temperature distribution in the laptop mother board during operating with one more new suggested thermal management system. The new suggested design consists of flat plate of copper material coated with graphene and attached to the microprocessor and work with only passive cooling technique without fan. Considerable drop in microprocessor temperature is obtained through the heat dissipation path suggested in the new design. The temperature drop in the new design is due to increasing of both surface area of heat transfer and thermal conductivity of the graphene. The proposed passive cooling solution has the advantages of fanless operation compared to the existing active cooling solutions such as the noise-free operation, lower energy consumption and higher reliability.

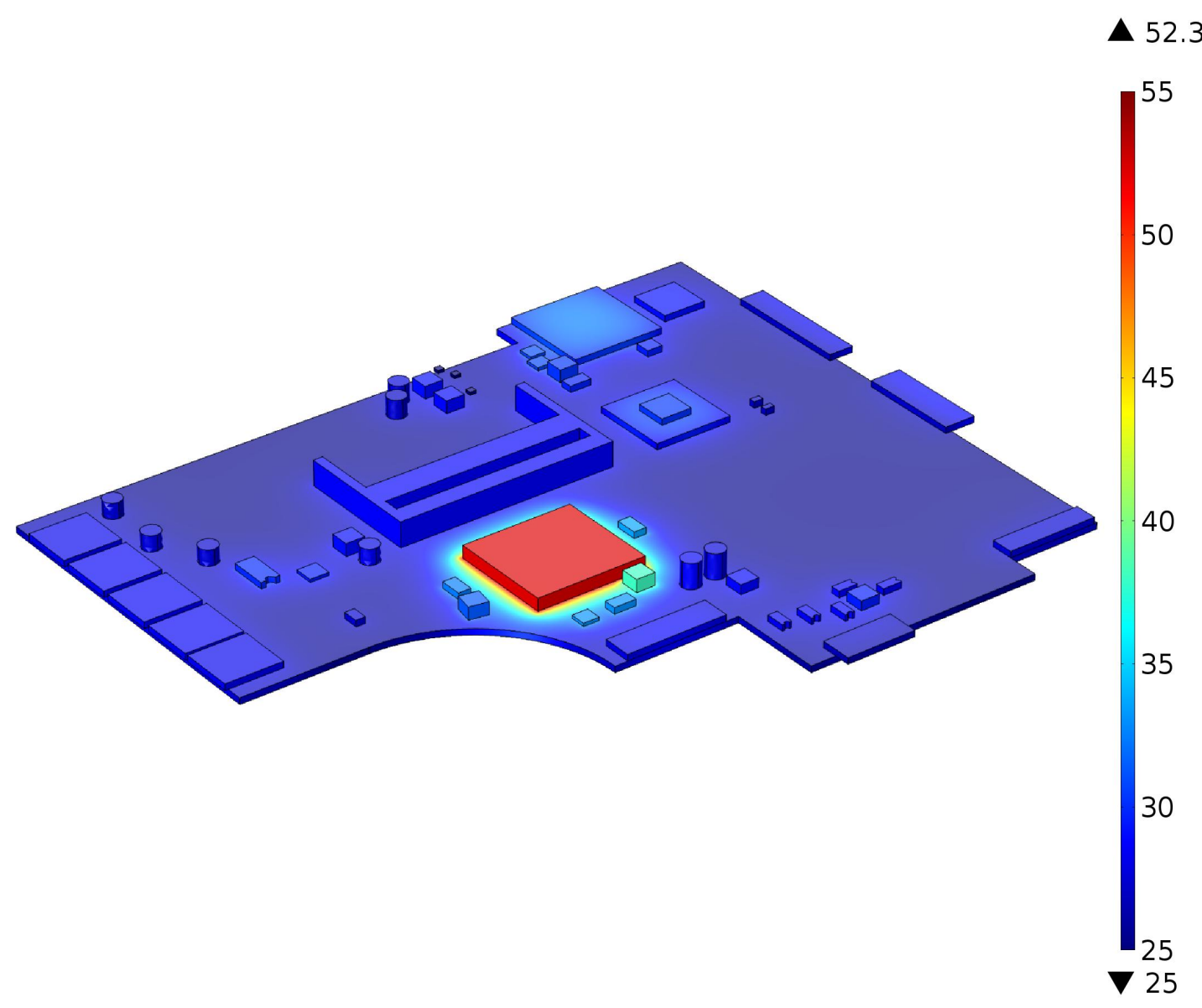

(a) laptop mother board without thermal management system (heat pipe). 
A 45.2

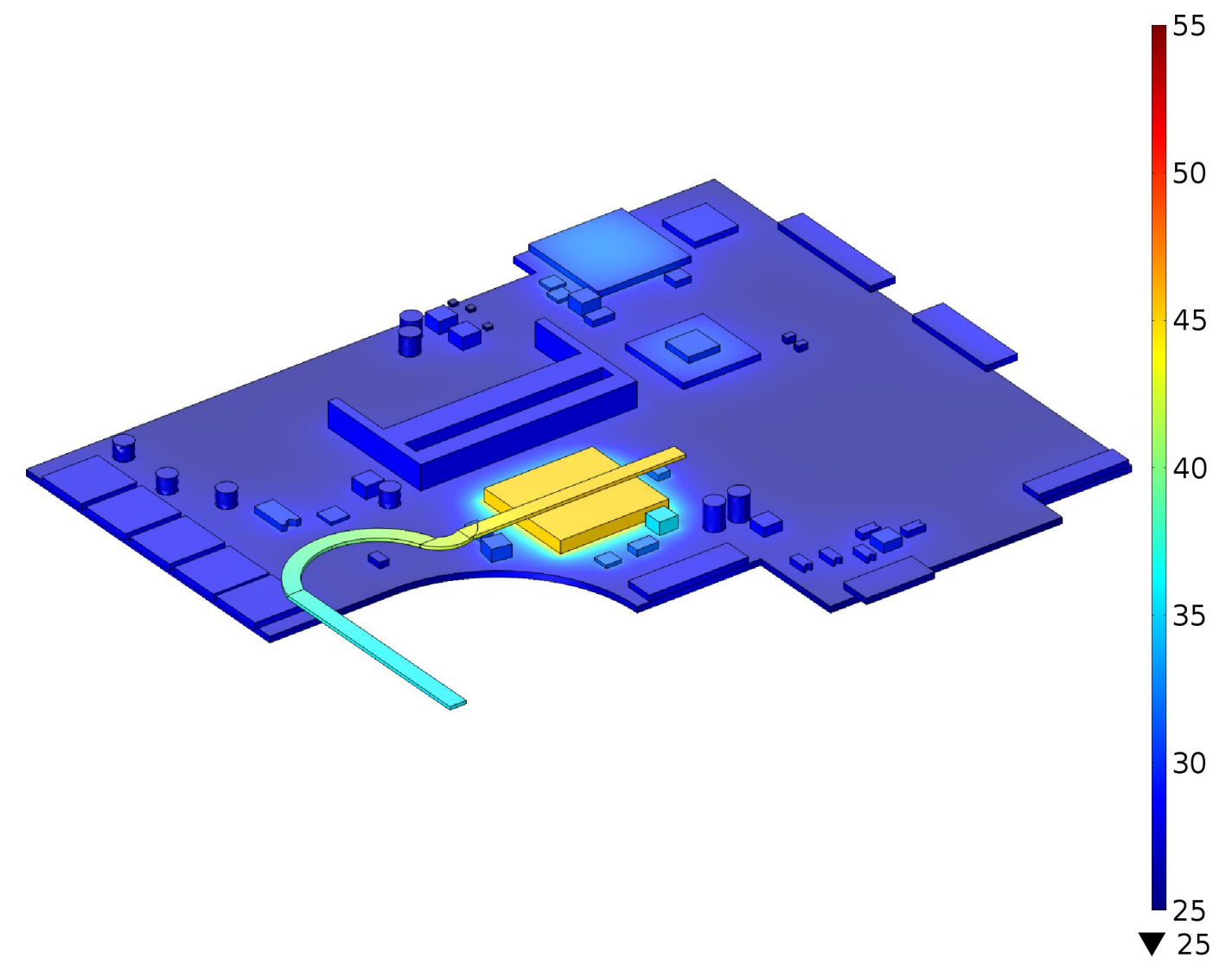

(b) laptop mother board with thermal management system (heat pipe).

Figure 4. Temperature distribution in the laptop mother board $\left[{ }^{\circ} \mathrm{C}\right]$ during operating without and with thermal management system (copper heat pipe). 


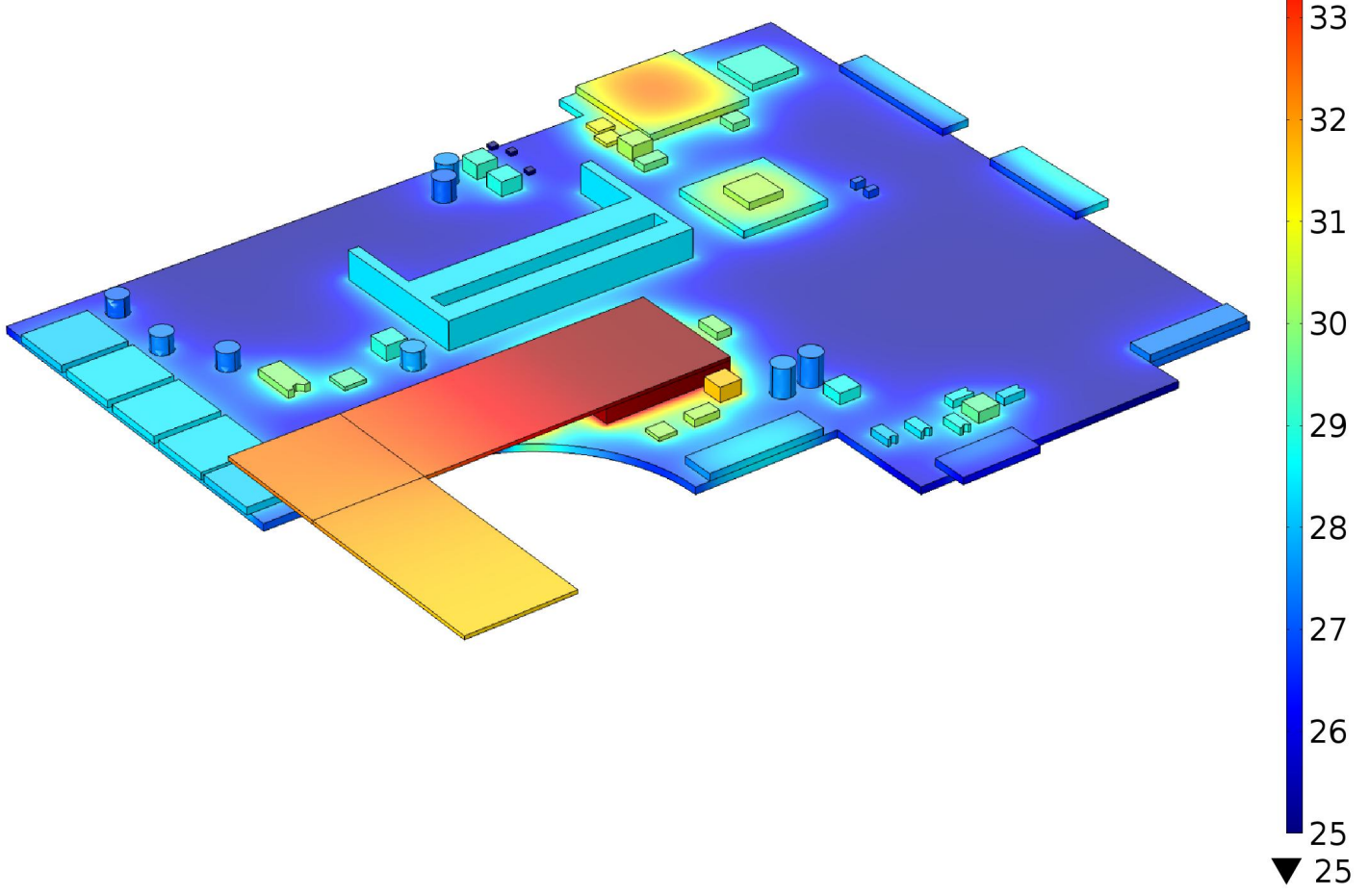

Figure 5. Temperature distribution in the laptop mother board $\left[{ }^{\circ} \mathrm{C}\right]$ during operating with new suggested thermal management system (flat plate of copper material coated with graphene and without use of fan).

\section{Conclusion}

Three dimensional steady state CFD model of a laptop motherboard has been presented. The model accounts for heat transfer for both natural convection and radiation to the ambient air temperature. The present CFD study allow accurate, rapid, physical modelling to make decisions on materials, components and layout beside power control feedback to achieve performance and target lifetime with reduced testing requirements. The model is shown to be able to: (1) understand the many interacting and heat transfer phenomena, which, very difficult or cannot be studied experimentally; (2) identify limiting steps and components; and (3) provide a computer-aided tool for design and optimization of future electronic cooling with efficient and economical solutions.

An alternative design for the cooling of laptop microprocessor using only passive cooling is proposed. The results showed that the assembled a thin plate of a copper material coated with a graphene and use it as a heat sinks with the microprocessor of the laptop providing an efficient and economical solution in thermal management. Finally, in conclusion this proposed passive cooling solution is better in heat dissipation and cooling the system when compared to the existing active cooling solutions. It also has additional advantages such as the noise-free operation, lower energy consumption and higher reliability. We hope this work may open the way for huge boost in the technology of electric cooling by innovative manufacturing techniques.

\section{References}

1. Yunus A. Çengel. Heat and Mass Transfer: A Practical Approach, chapter 15: Cooling of Electronic Equipment; McGraw-Hill Higher Education: ISBN: 0073129305, 2007; pp. 1. 
2. Lienard, J.H.. A Heat Transfer Textbook; MIT Press: USA, 2014; pp. 1.

3. Remsburg, Ralph. Thermal design of electronic equipment; CRC Press: ISBN 0-8493-0082-7, USA, $2001 ;$ pp. 1.

4. Jagadish Thammanna, Ambuj Srivastav. Thermal management in electronic equipment; HCL Technologies: USA, 2010; pp. 1.

5. Enrico Dallago; Thermal characterization of compact electronic systems: a portable PC as a study case. IEEE Transactions on power electronics 2002, 17, 187-195.

6. Peter Rodgers; The need for electronics thermal design practices to embrace sustainability. Electronics cooling 2014, September 2014, 2.

7. R.Mohan and P.Govindarajan; Thermal Analysis of CPU with variable Heat Sink Base Plate Thickness using CFD. International Journal of the Computer, the Internet and Management2010, 18, 27-36.

8. Ilker Tari, and Fidan Seza Yalcin; CFD Analyses of a Notebook Computer Thermal Management System and a Proposed Passive Cooling Alternative. IEEE Transactions on Components and Packaging Technologies2010, 33, 443452.

9. N.Hariharan, A.S. Manirathnam, S.Vellingiri, R.S. Mohankumar; CFD thermal analysis on laptop cooling system using loop heat pipe technology. International Journal of Research in Engineering and Technology 2014, 3, 676-682.

10. Manish Sharma, B. B. Saxena, K. K. Thakur, Sanjay Kumbhare; CFD analysis of a laptop thermal management system. International Journal of Advanced Engineering Technology 2015, 6, 29-31.

11. Man-In Baek, Jung-Mi Lee. Thermal Design for NOTEBOOK PC by using thermal analysis. LG Electronics Inc., LG Production Research center, white paper.

12. Eric Pop, Vikas Varshney, Ajit K. Roy; Thermal properties of graphene: Fundamentals and applications. MRS BULLETIN 2012, 37, 1273-1281.

13. Goki Eda, Manish Chhowalla; Graphene-based composite thin films for electronics. Nano Letter 2009, 9, 814-818.

14. Vivek Goya, Alexander A. Balandin; Thermal properties of the hybrid graphene-metal nano-micro-composites: Applications in thermal interface materials. Appl.Phys.Lett. 2012, 100, 073113.

15. Maher A.R. Sasiq Al-Baghdadi. Applications of Computational Fluid Dynamics and Finite Element Methods in Engineering Education; International Energy and Environment Foundation: ISBN-9781512122428, 2015 ; pp. 1.

\section{Keywords}

Electronic cooling; Portable computer; Thermal analysis; Heat pipe; CFD; Graphene

(c) 2020 by the author(s). Distribute under a Creative Commans CC BY license 